\title{
Cent ans de rayons cosmiques
}

Kumiko Kotera ${ }^{(1,2)}$ (kotera@iap.fr)

(1) Institut d'Astrophysique de Paris, CNRS UMR 7095, UPMC, 98 bis bd Arago, 75014 Paris

(2) California Institute of Technology, 1200 E California Blvd, Mailcode 350-17, Pasadena CA 91125, USA.

Il y a cent ans avait lieu ce que l'on pourrait appeler

la première expérience

physique spatiale concluante :

Victor Hess embarquait

un électroscope à bord

d'un ballon et démontrait

qu'un flux de particules

extraterrestres nous bombarde

continuellement. Ce flux

fut baptisé le rayonnement

cosmique.

Depuis leur découverte,

les rayons cosmiques tour

à tour nous intriguent

et éclairent nos pas dans

la physique et I'astrophysique

modernes : ils ont permis

l'émergence de la physique

des particules, et la question

de leur origine reste encore

aujourd'hui I'un des plus grands

mystères de l'astrophysique.

Remerciements

Je remercie Jim Cronin pour sa relecture attentive, et pour m'avoir généreusement prêté ses cours sur l'histoire des rayons cosmiques, dont je me suis inspirée.
Tout a commencé dans les années 1900 , lorsque les physiciens décidèrent de s'interroger sur le fait que leurs électroscopes ultra-stables, conçus par Theodore Wulf pour étudier la radioactivité, se déchargeaient spontanément. On pensait à cette époque que des substances radioactives émanant de la Terre ionisaient l'air, ce qui déchargeait les appareils. On s'attendait ainsi à ce que le taux de déchargement spontané diminue en montant en altitude. Plusieurs expériences en ballon avec des électroscopes embarqués furent menées. Entre avril et août 1912, Victor Hess entreprit sept vols en ballon et mesura clairement que contrairement aux attentes, le taux de déchargement augmentait avec l'altitude. Il exclut le fait que l'ionisation soit due au Soleil, en effectuant des vols pendant la nuit et même au cours d'une éclipse solaire. Hess conclut alors : "la seule manière d'interpréter mes résultats expérimentaux fut d'admettre l'existence d'un rayonnement très pénétrant, d'une nature encore inconnue, venant principalement d'en haut, très probablement d'origine extraterrestre ", et appela ce " rayonnement d'en haut " Hohenstrahlung.

En 1925, Robert Millikan, qui avait regardé cette origine extraterrestre d'un œil sceptique, finit par en être convaincu, en menant lui-même des mesures d'ionisation dans des lacs, à différentes profondeurs. Il rebaptisa le Hohenstrahlung rayonnement cosmique. Millikan croyait alors (et ce, jusqu'à la fin de sa vie) que le rayonnement cosmique était constitué de lumière, d'où le terme " rayonnement ". C'est cette nomenclature qui nous est restée, bien que nous sachions à présent que ces particules cosmiques ne sont pas des photons, mais des noyaux atomiques.

Cette question de la nature des rayons cosmiques donna lieu à un débat acharné opposant notamment Robert Millikan et Arthur Compton. Millikan était persuadé de la nature photonique du rayonnement,

\section{Ëbe New Hork Ëimes}

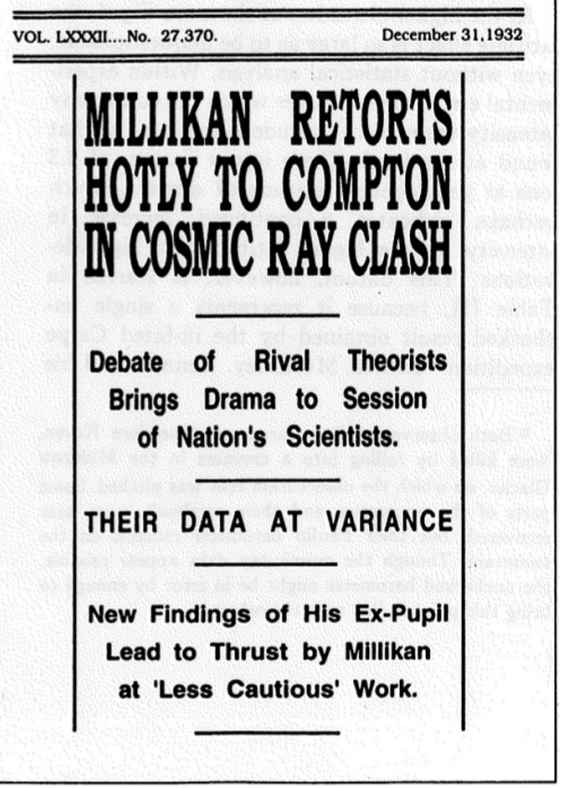

1. La une du New York Times du 31 décembre 1932.

alors que Compton stipulait que les rayons cosmiques étaient des particules chargées électriquement. Leur débat atteignit une telle virulence à l'occasion de la réunion de Noël de l'Association Américaine pour l'Avancement des Sciences de 1932, que le New York Times en fit sa une le 31 décembre (fig. 1).

Au cours des années 30, de nombreuses expériences démontrèrent que les rayons cosmiques sont des particules chargées de façon positive pour plus de $90 \%$ d'entre eux. Tout d'abord, les physiciens Clay et Compton entreprirent de grands périples en mer et mirent en évidence la déviation des rayons cosmiques par le champ magnétique terrestre. À différents endroits du globe, ces expérimentateurs purent mesurer que le taux d'ionisation au niveau de la mer augmentait avec la latitude, ce qui confirmait le caractère chargé des particules (fig. 2). Puis, entre 1933 et 1934, trois études 


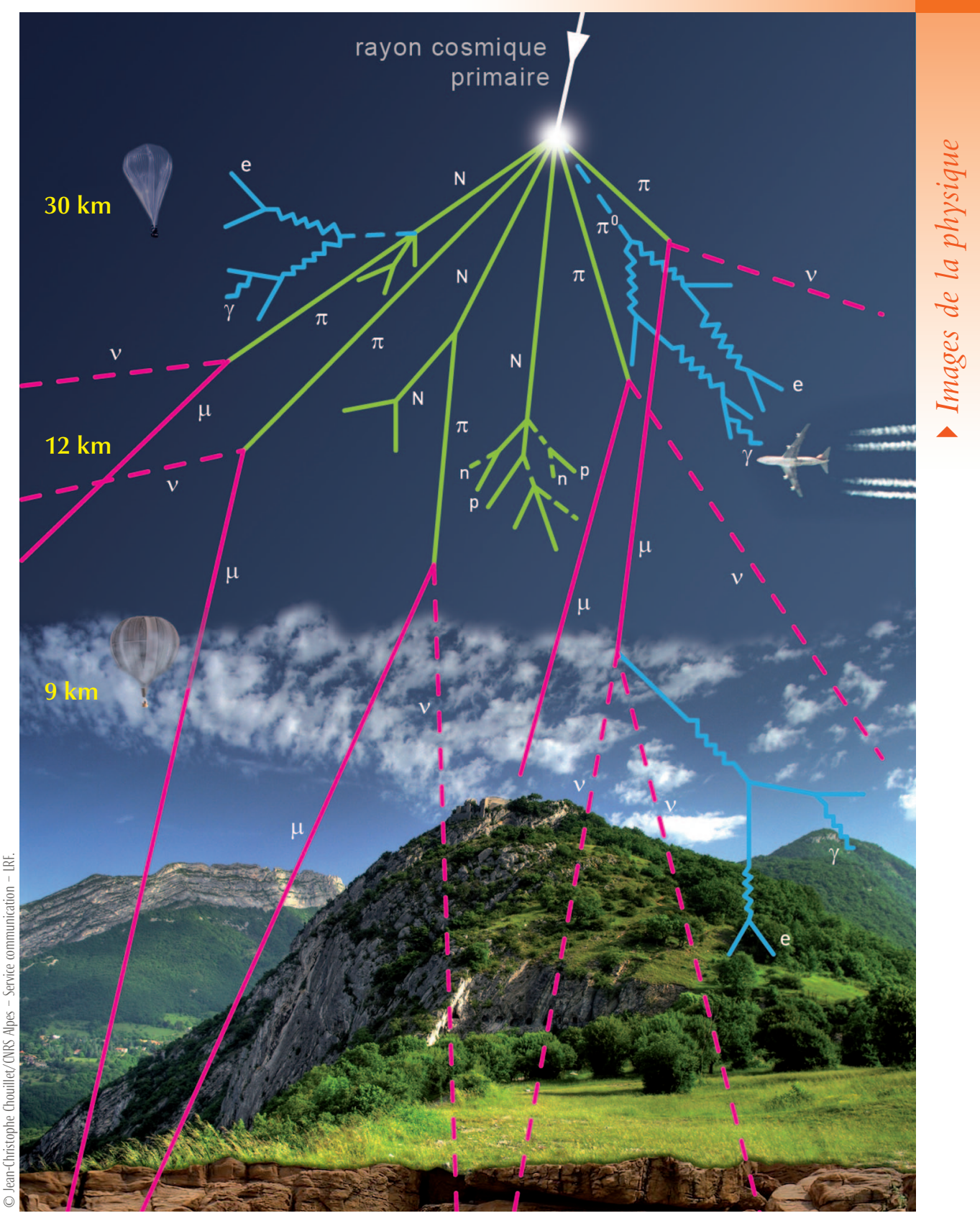

indépendantes, menées par Alvarez et Compton, par Johnson, et par Rossi, montrèrent que le flux de rayons cosmiques en provenance de l'ouest était plus important que celui de l'est. Cette mesure de l'effet " est-ouest ", prédit plus tôt par Rossi et par Lemaitre et Vallarta, démontrait que les rayons cosmiques portaient une charge positive. En effet, la structure du champ magnétique terrestre est telle qu'un faisceau de particules qui parvient sur la Terre selon une trajectoire parallèle au plan équatorial doit être dévié vers l'ouest pour des charges positives et vers l'est pour des charges négatives.

$\mathrm{Au}$ cours de ses expériences sur l'effet est-ouest, Bruno Rossi remarqua que des signaux arrivaient en coïncidence sur les compteurs Geiger dont il se servait pour mesurer le taux de rayons cosmiques, qui n'étaient placés qu'à quelques pas de distance. Cinq ans plus tard, en 1939, Pierre Auger étudiait intensivement (et extensivement !) cet effet. À l'aide d'un dispositif couvrant une superficie de l'ordre du kilomètre carré, au col du Jungfraujoch, à 3500 mètres d'altitude en Suisse, Auger découvrit, par un système de coïncidences temporelles, le phénomène de gerbe cosmique. Ces gerbes sont initiées par des rayons cosmiques de haute énergie, dans les hautes couches de l'atmosphère terrestre, à plus de 20 kilomètres d'altitude (fig. 3). Par interaction avec les molécules de l'atmosphère, elles produisent une cascade de particules secondaires qui atteignent le sol en gerbes composées principalement d'électrons, de photons et de muons. La pluie de particules peut alors se déployer sur plusieurs kilomètres, et ce sont ces gerbes que Auger avait détectées avec ses compteurs Geiger. En mesurant leurs caractéristiques, Auger avait pu conclure que l'énergie des rayons cosmiques primaires pouvait dépasser $10^{15} \mathrm{eV}^{(1)}$. Aujourd'hui, les plus grands accélérateurs de particules peuvent tout juste atteindre $10^{14} \mathrm{eV}$.

3. Développement d'une gerbe atmosphérique (voir texte). En vert, les interactions hadroniques et leurs produits; en rose, les produits de la désintégration des pions; en bleu, les produits électromagnétiques. Les altitudes auxquelles se développent les différentes parties de la gerbe sont indiquées sur la gauche.

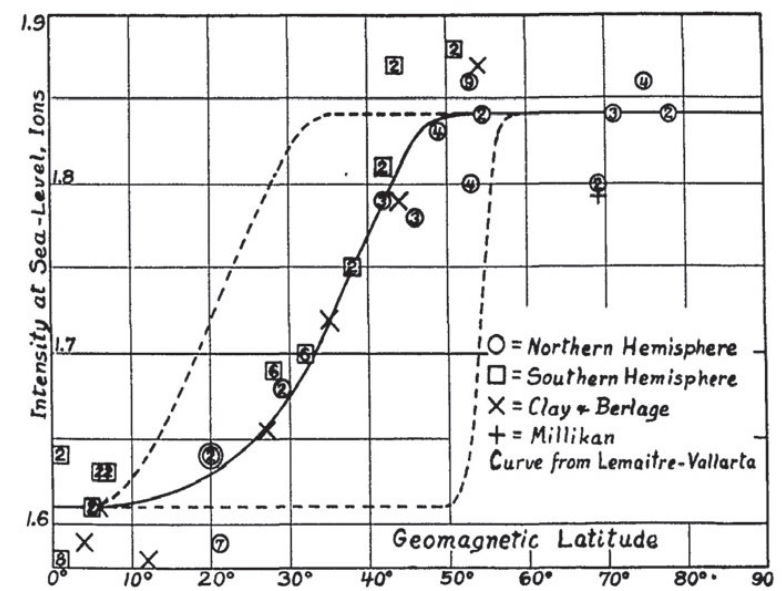

2. Mise en évidence de la nature chargée des rayons cosmiques arrivant dans la haute atmosphère terrestre. Les mesures expérimentales de Compton (cercles et carrés) et celles de Clay et Berlage (croix) sont superposées aux courbes de Lemaitre et Vallarta, qui prédisent la variation du taux d'ionisation de l'atmosphère en fonction de la latitude, pour un flux de particules chargées se propageant dans le champ magnétique terrestre (trait plein). Si les particules n'étaient pas chargées, une telle variation ne serait pas observée. (A. H. Compton, Phys. Rev. 43 (1933) 387). 

des particules

Ces particules, dotées d'énergies colossales, étaient une aubaine pour sonder la matière et, à partir du début des années 1930, les rayons cosmiques devinrent un outil indispensable pour la physique des particules. En étudiant les gerbes de rayons cosmiques à l'aide d'une chambre de Wilson (voir encadré 1 et fig. 4), les physiciens purent mettre en évidence de nombreuses particules élémentaires comme le positron, le muon et le kaon, entre les années 1920 et 1950. Ces découvertes donnèrent lieu à une série de prix Nobel : notamment, le physicien Carl Anderson fut récompensé en 1936 pour la découverte du positron.

Après la guerre fut mise en place une nouvelle technique de détection basée sur des émulsions photographiques, où les particules chargées laissaient des traces qui pouvaient s'examiner en détail au microscope. Le physicien italien Giuseppe Occhialini se servit de cette technique d'abord au Pic du Midi, puis dans les Andes boliviennes : à haute altitude, on avait accès à des particules de plus haute énergie. L'analyse des émulsions ainsi obtenues conduisit Occhialini et le physicien anglais Cecil Powell à identifier une particule de masse intermédiaire, dont l'existence avait été postulée en 1935 par le Japonais Hideki Yukawa : le pion. Yukawa reçut le prix Nobel en 1949, puis Powell en 1950. Le collaborateur anglais de Occhialini, Patrick Blackett, reçut lui aussi le Nobel pour l'ensemble de ses travaux. Seul Occhialini restait non couronné malgré ses contributions importantes: ses vues politiques trop progressistes en ces temps de guerre froide en sont probablement la cause.

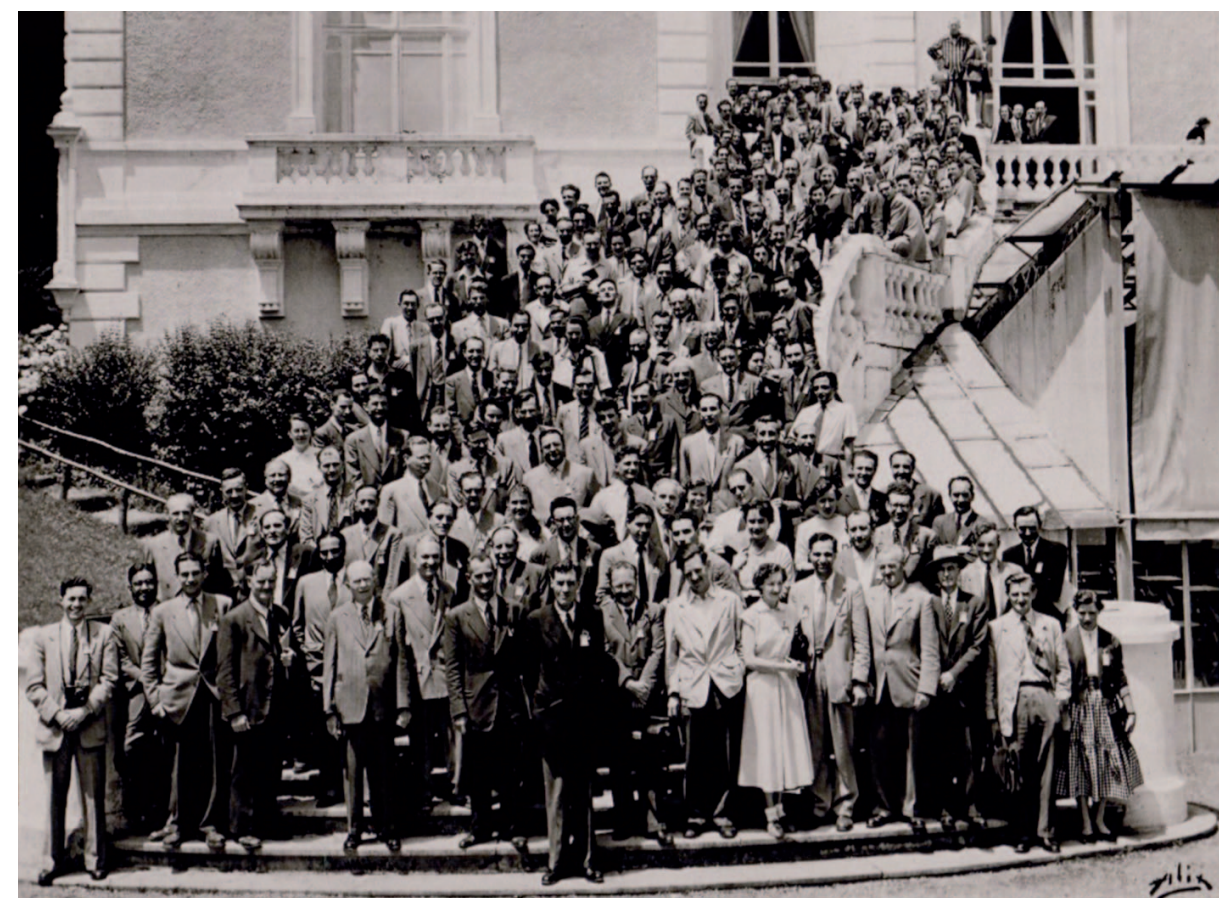

5. Photographie officielle du congrès de Bagnères-de-Bigorre de 1953. Leprince-Ringuet et Blackett, en veste noire, se tiennent au centre du premier rang. Extrait de The 1953 Cosmic Ray Conference at Bagnères de Bigorre de James W. Cronin (2011).

En juillet 1953 eut lieu le Congrès international sur le rayonnement cosmique à Bagnères-de-Bigorre (Hautes-Pyrénées). Les participants de ce congrès, organisé par Louis Leprince-Ringuet et Blackett, s'en rappellent tous comme de la meilleure conférence de leur vie. Elle a regroupé la plupart des grands physiciens de l'époque, qui ont rassemblé leurs découvertes et pu mettre en place les fondements de la physique subatomique. Quasiment toutes les conclusions tirées à cette conférence se sont plus tard révélées être correctes. Le congrès de Bagnères-de-Bigorre a par ailleurs marqué la transition de la physique des particules, de l'étude des rayons cosmiques à l'ère des accélérateurs de particules, qui entraient peu à peu en service en Europe, aux États-Unis et en Union Soviétique.

\section{Chambre de Wilson ou chambre à brouillard} encadré 1

La chambre de Wilson, appelée aussi chambre à brouillard, est une enceinte emplie d'une vapeur d'eau ou d'alcool, maintenue à une température très proche de son point de rosée. Lorsqu'une particule chargée traverse cette chambre, elle ionise les atomes de gaz sur son parcours. Les ions ainsi produits jouent le rôle de germe pour la condensation, et des gouttelettes se forment le long de la trajectoire de la particule. Les traînées qui se dessinent ont une forme caractéristique qui dépend de la particule ionisante. Lorsqu'un champ magnétique est appliqué dans la chambre, la trajectoire des particules chargées se courbe, et les charges positives et négatives suivent des directions opposées.

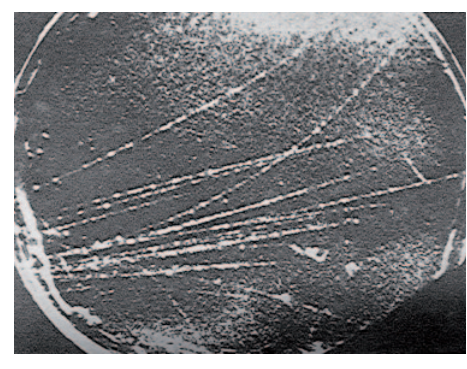

4. L'une des premières photographies de gerbes de rayons cosmiques dans une chambre de Wilson, obtenue par Blackett et Occhialini (Proc. Roy. Soc. London A 139 (1933) 699).
L'astrophysique du rayonnement cosmique : une autre fenêtre sur l'Univers

Vers cette même époque, quand les physiciens des particules délaissaient les rayons cosmiques pour leurs accélérateurs, les astrophysiciens commençaient enfin à s'y intéresser en tant que messagers de l'Univers. Le message des rayons cosmiques est cependant difficile à interpréter. En effet, des champs magnétiques terrestre, galactiques et extragalactiques dévient leur trajectoire au cours de leur périple jusqu'à la Terre, et rendent ardue l'identification de leurs sources.

En 1950, les radiotélescopes permirent de mesurer qu'une fraction importante du rayonnement radiocosmique provenait d'une émission synchrotron, ce qui indiquait la présence d'électrons relativistes dans toute notre Galaxie. On put ainsi cartographier l'abondance des électrons du rayonnement cosmique et commencer à travailler sur la modélisation de son transport dans la Galaxie. Cependant, les électrons constituent à peine $1 \%$ du rayonnement cosmique, le reste étant constitué de noyaux. En 1948, des ballons sondes des universités du Minnesota et de Rochester rapportèrent en effet la présence de noyaux lourds dans le rayonnement cosmique primaire. Il fut bientôt établi que pratiquement tous les éléments entre l'hydrogène et le fer étaient présents dans le rayonnement cosmique dans la haute atmosphère. 
Quelles sources pouvaient bien accélérer des noyaux à des énergies de l'ordre de $10^{15} \mathrm{eV}$, et par quel mécanisme ? Dès 1934, Baade et Zwicky proposaient un scénario d'accélération de particules basé sur les explosions de supernovæ. Plus tard, en 1949, Fermi posa les fondements des théories modernes de l'accélération des rayons cosmiques et de leur transport, en les considérant comme un gaz de particules relativistes chargées en mouvement dans le milieu interstellaire. Ses théories sur l'accélération portent aujourd'hui son nom.

Pour identifier les sources des rayons cosmiques galactiques, on eut recours à leurs messagers secondaires, les rayons gamma qu'ils produisent en interagissant avec les atomes du milieu interstellaire, au sein de notre Galaxie. Les rayons gamma, non chargés, peuvent se propager en ligne droite et peut-être nous indiquer les sources des protons et autres noyaux accélérés qui les ont produits. Dans la gamme d'énergie qui intéressait alors les astrophysiciens (entre $10^{8}$ et $10^{10} \mathrm{eV}$ ), les rayons gamma, arrêtés par l'atmosphère, ne peuvent être détectés que dans l'espace.

Dans les années 1960 justement, les expériences spatiales florissaient du fait de la compétition avec le bloc soviétique. De 1964 à 1966, les satellites soviétiques PROTON déterminèrent l'intensité et le spectre des rayons cosmiques jusqu'à $10^{12} \mathrm{eV}$. À la même époque, Occhialini fondait l'ESRO (2), ancêtre de l'Agence Spatiale Européenne, et lançait en 1969 la réalisation d'un satellite entièrement dédié à l'astronomie des rayons gamma de haute énergie : Cos B. Entre 1975 et 1982, Cos B fournit aux astrophysiciens des cartes du ciel gamma, dont les structures apparentes rappelaient fortement celles des cartes établies avec les observations radio. Les rayons cosmiques, électrons comme noyaux, emplissent donc la Galaxie et présentent une surdensité dans des régions centrales de la Voie lactée. L'observatoire à rayons gamma américain Compton prit le relais de Cos B de 1991 à 2000, afin d'étudier les conditions d'accélération et de propagation du rayonnement cosmique. Les théories comme les observations semblaient indiquer que les explosions de supernovæ étaient à l'origine des rayons cosmiques galactiques. Aujourd'hui, le satellite Fermi a pris le relais et, à encore plus haute énergie, les expériences au sol HESS et HESS-2 mesurent les gerbes induites par les rayons gamma.

\section{Le mystère des ultra-hautes énergies}

La théorie de l'origine des rayons cosmiques galactiques n'est toujours pas clairement établie aujourd'hui. Dans les années 1960, un autre mystère, encore plus intrigant, venait s'ajouter à la liste déjà longue des questions posées par les rayons cosmiques. En mesurant des gerbes atmosphériques au sol à l'aide d'une grille de détecteurs installée au Nouveau-Mexique - Volcano Ranch -, John Linsley et ses collaborateurs découvrirent des particules dotées d'énergies qui étaient quelques milliers de fois supérieures à celles prédites par Fermi. Ces particules nous parviennent en effet avec des énergies supérieures à $10^{20} \mathrm{eV}$ (des dizaines de joules), l'énergie d'une pierre lancée à la main, confinée dans une particule subatomique. Ces rayons cosmiques, dits de "ultra-haute énergie ", constituent l'une des plus grandes énigmes de l'astrophysique actuelle.

Quelles sources peuvent accélérer des particules à de telles énergies? Comment? Seuls les objets les plus puissants de l'Univers pourraient potentiellement accélérer ces rayons cosmiques. Les candidats prometteurs sont (fig. 6) : certains noyaux actifs de galaxie - des trous noirs supermassifs au centre de certaines galaxies, qui peuvent produire des jets de matière et de rayonnement extrêmement puissants -, les sursauts gamma - provoqués par les explosions d'étoiles les plus puissantes de l'Univers -, et les pulsars - des étoiles compactes fortement magnétisées et en rotation rapide. À ces énergies, la déviation due aux champs magnétiques est très petite. Pourtant, aucune source puissante n'est observée dans le ciel dans la direction d'arrivée de ces particules, et le mystère de leur provenance reste entier.

Alors que les particules d'énergie $10^{20} \mathrm{eV}$ étaient mesurées pour la première fois, Arno Penzias et Robert Wilson faisaient une autre découverte remarquable : celle $\mathrm{du}$ fond diffus cosmologique. L'Univers baigne dans un bain de photons de température moyenne 2,7 K. Kenneth Greisen, Vadem Kuzmin et Georgi Zatsepin, en recoupant ces informations, prédirent que les rayons cosmiques de ultra-haute énergie devaient interagir avec les photons du fond diffus et perdre leur énergie au fur et à mesure de leur propagation. Ainsi, les particules d'énergie supérieure à $610^{19} \mathrm{eV}$ ne pourraient pas nous parvenir depuis des
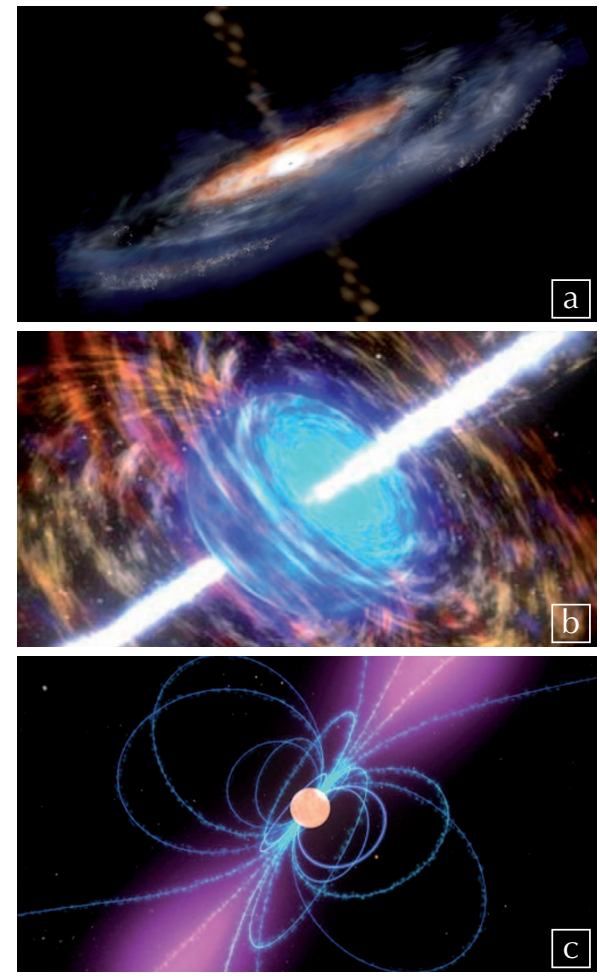

6. Trois sources possibles de rayons cosmiques de ultra-haute énergie.

(a) Des quantités colossales d'énergie sont libérées lorsque la matière tombe sur un trou noir supermassif au centre d'un noyau actif de galaxie. (Vue d'artiste Aurore Simonnet, NASA, E/P0, Sonoma State University.) (b) Explosion d'un sursaut gamma, produisant un jet d'une luminosité extrême. (Vue d'artiste : NASA/ SkyWorks Digital.)

(c) Des nuages de particules chargées se déplacent le long des lignes de champ magnétique d'un pulsar (en bleu) et créent un faisceau de rayons gamma (en violet) tournant à la manière d'un phare de marine. (Vue d'artiste: NASA/Goddard Space Flight Center Conceptual Image Lab.)

distances cosmologiques. Si les sources des rayons cosmiques de ultra-haute énergie sont situées à des distances intergalactiques, leur spectre en énergie devrait donc s'effondrer au-dessus de $610^{19} \mathrm{eV}$ : c'est la " coupure GZK ", prédite par Greisen, Zatsepin et Kuzmin en 1966. Il semble que les données les plus récentes indiquent l'existence de cette coupure (voir encadré 2). On ne peut cependant exclure la possibilité que le décrochement observé soit plutôt la signature d'une énergie d'accélération maximale à la source : il n'y aurait plus de particules au-delà d'une certaine énergie, simplement parce que les sources ne peuvent pas les produire. Avec plus de statistique, il sera possible de mesurer la forme exacte de cette coupure et ainsi de comprendre sa nature, pour clarifier l'origine des rayons cosmiques de ultra-haute énergie. 


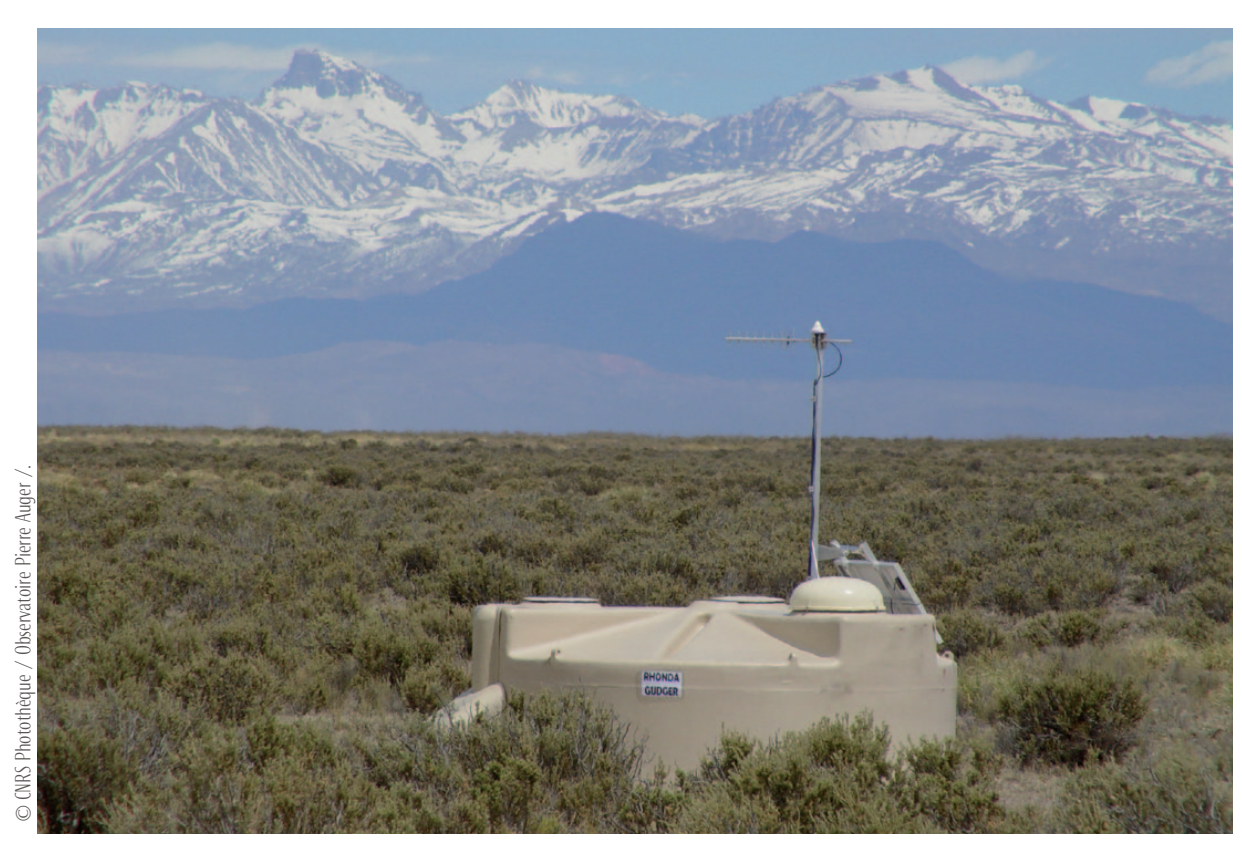

L'observatoire Pierre Auger, dans la pampa au pied des Andes, à $370 \mathrm{~km}$ au sud de la ville de Mendoza en Argentine. Vue d'une cuve de détection des particules secondaires produites dans les gerbes atmosphériques.

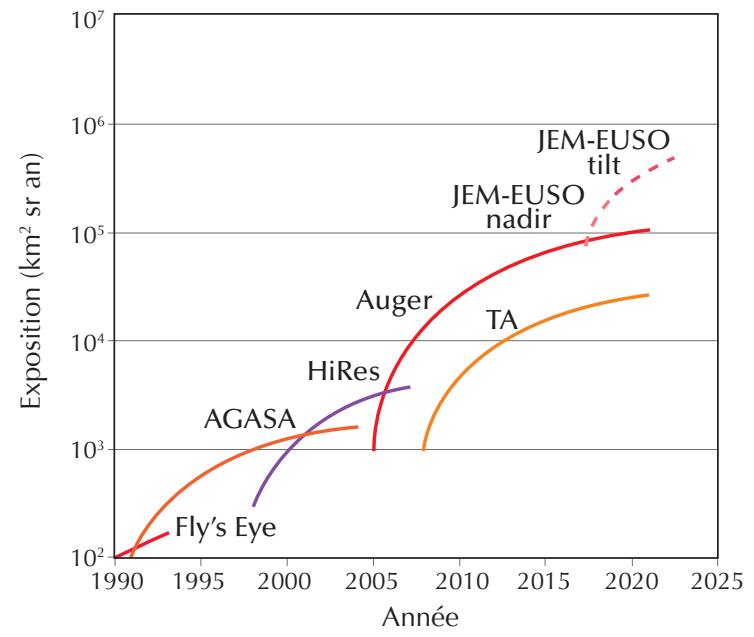

\section{$\gg>$}

Accumuler de fortes statistiques d'événements n'est pas une tâche aisée. En effet, le flux des rayons cosmiques de ultrahaute énergie est très faible : on reçoit sur Terre environ une particule de ce type par kilomètre carré par siècle. Des observatoires couvrant des terrains de plus en plus grands ont donc été construits (fig. 7). Le plus grand détecteur à ce jour est l'observatoire Pierre Auger, qui couvre $3000 \mathrm{~km}^{2}$ de pampa, dans la province de Mendoza en Argentine. Cet observatoire international est constitué d'une grille de cuves d'eau posées au sol, espacées de 1,5 km, capables de détecter les particules secondaires produites dans les gerbes atmosphériques. De surcroît, quatre sites de télescopes ont été installés, afin d'observer directement la faible lumière fluorescente émise par le développement des gerbes dans le ciel. L'autre observatoire aujourd'hui en activité est le Telescope Array (TA). Ce projet japonais et américain est de taille plus modeste $\left(762 \mathrm{~km}^{2}\right)$, mais il constitue le plus grand détecteur de l'hémisphère Nord.

7. Évolution dans le temps de l'exposition pour les expériences passées, actuelles et en projet : Fly's Eye, AGASA, HiRes, I'Observatoire Pierre Auger, Telescope Array (TA). Exposition estimée pour le projet JEM-EUSO pour un lancement en 2017. En ordonnée : produit de la surface de collection par la durée d'exposition et l'angle solide visible, modulée par les limitations physiques instrumentales, en unités de kilomètres carrés.stéradians.an. (D'après Kotera et Olinto, ARAA, 2011.)
À ce jour, les scientifiques ont collecté, à l'aide de tous ces instruments, une centaine de rayons cosmiques de ultra-haute énergie, et nous avons déjà pu éliminer plusieurs sources candidates. Grâce aux données sur le spectre en énergie, les directions d'arrivée et la composition des particules observées, nous tenons des pistes sérieuses sur l'origine de ces rayonnements extrêmement énergétiques (fig. 6). La statistique actuelle n'est pourtant pas encore suffisante pour avoir une vision complète et précise, et identifier la ou les sources.

\section{La jambe cosmique : genou, cheville, coupure GZK}

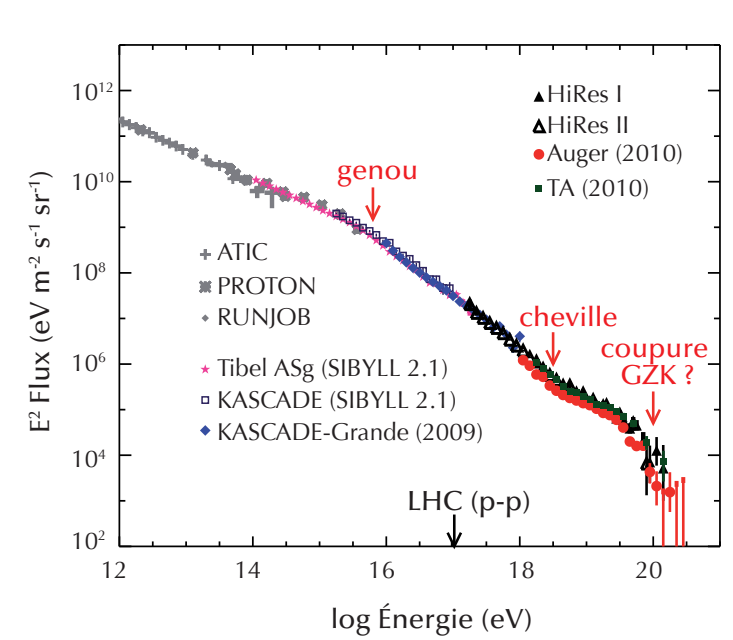

La figure ci-contre représente le flux ou spectre de rayons cosmiques de haute énergie reçu sur Terre (multiplié par l'énergie de chaque particule au carré pour faire apparaître plus clairement les cassures) en fonction de leur énergie. Le spectre représenté ici résulte de la compilation de plusieurs expériences en ballon et par satellite, et d'expériences à plus haute énergie utilisant des grilles de détecteurs au sol. Les gerbes atmosphériques mesurées sont analysées avec des modèles $d$ 'interaction hadronique différents. La flèche " LHC » indique l'énergie dans le centre de masse à laquelle les interactions proton-proton peuvent être testées avec l'accélérateur terrestre LHC.

Avec un peu d'imagination, on peut voir dans cette loi de puissance, qui présente plusieurs cassures, la forme d'une jambe : un premier raidissement vers $10^{16} \mathrm{eV}$ est appelé le " genou », une atténuation de la pente vers $10^{18,5} \mathrm{eV}$ s'apparente à la " cheville », et la coupure au-delà de $610^{19} \mathrm{eV}$ est peut-être la coupure GZK prédite en 1966 par Greisen, Zatsepin et Kuzmin, qui serait provoquée par I'interaction des photons du fond diffus cosmologique avec les rayons cosmiques extragalactiques (voir texte). 
Le prochain observatoire de rayons cosmiques de ultra-haute énergie en projet est la mission JEM-EUSO ${ }^{(3)}$, qui devrait être installée sur la station spatiale internationale (fig. 8). Son objectif est d'observer le développement des gerbes atmosphériques depuis l'espace, et ainsi de balayer une surface au sol 100 fois supérieure à la surface de collection de l'Observatoire Pierre Auger ${ }^{(4)}$. Il est envisagé de basculer le télescope de son axe perpendiculaire au sol (mode "nadir") à un angle de quelques dizaines de degrés (mode "tilt") après quelques années. Ce mode "tilt" entrainerait une perte en résolution, mais permettrait d'augmenter encore la surface balayée au sol, et ainsi de collecter encore plus de rayons cosmiques de ultra-haute énergie.

\section{0 ans plus tard...}

En cent ans, les rayons cosmiques ont lancé la physique des particules, ouvert des fenêtres d'observation sur l'Univers, intrigué tout un monde de physiciens et d'astronomes avec leurs énergies fabuleuses et leurs trajectoires énigmatiques.

Cependant, il y a encore seulement une dizaine d'années, le nombre très restreint de données et le risque que les champs magnétiques ne brouillent entièrement les directions d'arrivée conduisaient beaucoup de scientifiques à voir en l'étude des rayons cosmiques un sujet ésotérique et spéculatif.

Le vent semble avoir tourné ces derniers temps : on prend conscience de l'importance de ces particules en tant que messagers privilégiés de l'Univers, et la communication avec les autres domaines de l'astrophysique s'établit. Les énigmes posées par ces particules concernent en effet l'ensemble de l'Univers : les champs magnétiques à toutes les échelles, les objets astrophysiques énergétiques, l'ensemble du rayonnement non thermique, et même la physique des neutrinos.

\section{Toujours de mèche avec la physique des particules}

L'astrophysique des rayons cosmiques de haute énergie a gardé un lien étroit avec la physique des particules. L'analyse des gerbes atmosphériques dépend en effet de modèles hadroniques qui peuvent être testés dans une certaine limite avec les accélérateurs de particules terrestres. Mais avec les rayons cosmiques de ultra-haute énergie, la nature nous permet d'accéder à un domaine d'énergie environ mille fois supérieur à ce que permet l'accélérateur de particules le plus puissant sur Terre, le LHC (Large Hadron Collider). Ceci est un atout pour la physique, mais également une difficulté pour les astrophysiciens. La physique des interactions dans ces gammes d'énergie extrême n'étant pas testée, nous ne pouvons qu'extrapoler les résultats des interactions hadroniques à plus basse énergie, et notre compréhension des gerbes atmosphériques générées par les rayons cosmiques de ultra-haute énergie reste donc incertaine. Les progrès en amont sur les rayons cosmiques permettront d'explorer la physique des particules à des énergies non encore reproductibles sur Terre ; réciproquement, les progrès en physique des particules nous offriront un meilleur outil pour interpréter nos observations des rayons cosmiques.

\section{Acteurs-clés de l'ère multimessager}

Nous sommes aujourd'hui à l'orée d'une ère nouvelle de l'astrophysique des hautes énergies : l'ère multimessager. Nos progrès sur la connaissance du fonctionnement des objets astrophysiques les plus puissants proviennent de l'analyse conjointe des informations fournies par différents messagers : par les photons bien sûr, à différentes longueurs d'ondes, mais aussi par les neutrinos, les ondes gravitationnelles et les rayons cosmiques. Ces derniers jouent un rôle central dans ce tableau, puisqu'ils sont à l'origine des autres émissions non thermiques. Certaines émissions radio, les émissions X et gamma, ainsi que la plupart des neutrinos, sont produits par les rayons cosmiques lors de leurs interactions avec la matière.

L'étude des rayons cosmiques est donc essentielle pour la compréhension de l'ensemble de l'astrophysique des hautes énergies, mais cette étude doit être menée de pair avec celle des autres messagers. Les instruments actuels et futurs tels que JEM-EUSO, Ice-Cube pour les neutrinos, LIGO pour les ondes gravitationnelles et $\mathrm{CTA}^{(5)}$ pour les rayons gamma devraient résoudre l'énigme des rayons cosmiques de ultra-haute énergie et apporter des réponses importantes sur l'ensemble des événements énergétiques qui peuplent notre Univers.

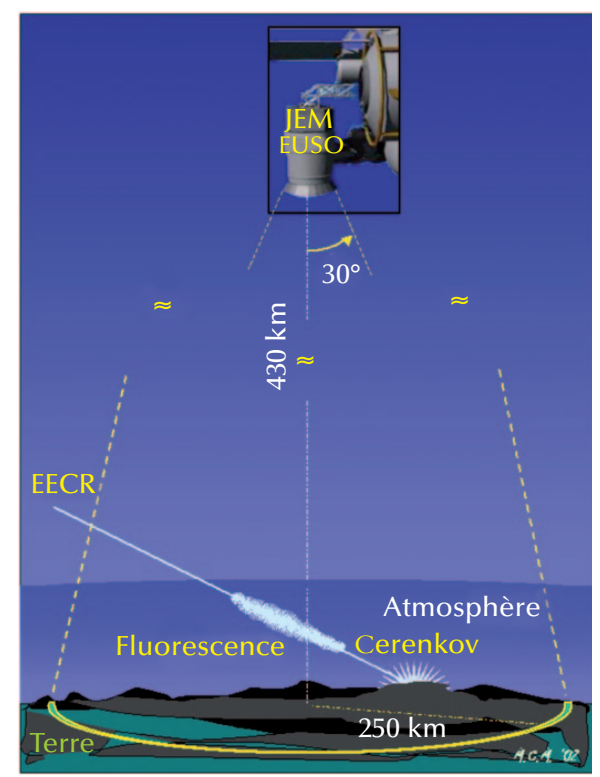

8. Le projet JEM-EUSO vise à observer depuis l'espace le rayonnement de fluorescence émis lors du développement des gerbes atmosphériques.

(1) Un électron-volt (eV) est l'énergie gagnée par un électron accéléré par une différence de potentiel de un volt.

(2) ESRO : Organisation Européenne de Recherches Spatiales

(3) JEM-EUSO : Extreme Universe Space Observatory attached to the Japanese Experiment Module.

(4) Dans les faits, malgré cette surface de collection bien supérieure, JEM-EUSO serait limité par un temps d'observation restreint, puisque le rayonnement de fluorescence des gerbes ne peut être observé que par nuit noire (20 à $30 \%$ du temps).

(5) CTA : Cherenkov Telescope Array.

\section{En savoir plus}

- T. K. Gaisser, Cosmic Rays and Particle Physics, Cambridge University Press (1990).

- École de Goutelas, Physique et astrophysique du rayonnement cosmique, ouvrage collectif dirigé par É. Parizot, A. Marcowith, V. Tatischeff, G. Pelletier et P. Salati, édité par l'Observatoire astronomique de Strasbourg (2004).

- J. W. Cronin, "The 1953 Cosmic Ray Conference at Bagneres de Bigorre: the Birth of Sub Atomic Physics", arXiv:1111.5338v1 (2011).

- K. Kotera et A. V. Olinto, "Astrophysics of Ultrahigh Energy Cosmic Rays”, Annual Review of Astronomy and Astrophysics, 49 (2011) 119.

- B. Degrange, « Nouvelles investigations sur l'origine des rayons cosmiques : 1. L'expérience HESS », Bulletin de la SFP n 151 (octobre 2005) 4-10. 